\title{
Wakefield radiation from the open end of an internally coated metallic tube
}

\author{
M. Ivanyan, ${ }^{*}$ A. Grigoryan, A. Tsakanian, and V. Tsakanov \\ CANDLE Synchrotron Research Institute, Acharyan 31, 0040 Yerevan, Armenia
}

(Received 25 February 2014; published 11 July 2014)

\begin{abstract}
In this paper the problem of radiation from the open end of a semi-infinite circular metallic waveguide with perfectly conducting walls and a thin internal low-conducting metal coating is considered. Electromagnetic fields are generated by the ultrarelativistic point charge moving along the axis of the waveguide. The far fields of radiation are obtained using the near-field to far-field recovery technique. The technique is extended for the nonmonochromatic waves. It is shown that the radiation has a narrow-band and narrow-directional character.
\end{abstract}

DOI: 10.1103/PhysRevSTAB.17.074701

PACS numbers: 41.60.-m

\section{INTRODUCTION}

Recently, a new traveling wave structure has been proposed [1]: an internally coated metallic pipe (ICMP), which has only a single slowly propagating (phase velocity is equal to the velocity of light) $\mathrm{TM}_{01}$ mode. Because of an absence of the high slowly propagating modes, the above structure could be effectively used for particle acceleration or generation of monochromatic radiation [2-5]. The experimental study of an ICMP type structure is currently under development at AREAL test facility [6]. The experimental study and exploitation of this radiation are supposed to extract the radiation from the open end of the ICMP structure. The knowledge about the spectral and angular distribution of extracted radiation is important for experimental verification, exploitation, and further development of the ICMP based devices.

In this paper the spatiotemporal and angular characteristics of the charged particle electromagnetic radiation from the open end of ICMP structure are studied. As shown in [1], the relativistic particle moving along the axis of the ICMP structure under certain conditions excites only the fundamental $\mathrm{TM}_{01}$ mode. The near-field to far-field recovery technique [7] is used to obtain radiated in ICMP wake fields transformation into the far zone from the open end of the waveguide. The technique is expanded for the non monochromatic waves [8]. The angular, space, and frequency patterns of the radiation are obtained.

\section{STATEMENT OF THE PROBLEM}

A semi-infinite circular waveguide with perfectly conducting walls internally covered by a thin metallic layer of

\footnotetext{
*Corresponding author. mikayel.ivanyan@gmail.com

Published by the American Physical Society under the terms of the Creative Commons Attribution 3.0 License. Further distribution of this work must maintain attribution to the author $(s)$ and the published article's title, journal citation, and DOI.
}

low conductivity $\sigma_{1}$ is considered (Fig. 1). The inner radius of the waveguide is $a$ and the thickness of the inner conductive layer is $d(d \ll a)$.

The electromagnetic properties of the internal layer is characterized by the dielectric permeability of the metal $\varepsilon_{1}=\varepsilon_{0}+j \sigma_{1} / \omega$, where $\varepsilon_{0}$ is the vacuum dielectric constant and $\omega$ is the frequency.

The electromagnetic wake fields are excited by the ultrarelativistic (with velocity $v=c, c$ being the speed of light) point charge $q$ moving along the axis of the waveguide. Time $t=0$ (Fig. 1) corresponds to the time when the charge reaches the open end of the waveguide. Because of the causality principle for an ultrarelativistic charge, the excited fields are behind the charge and for time $t \leq 0$ (position 1 in Fig. 1) the excited wake field coincides with the wake fields excited by charge in an infinitely long tube. The infinitely long ICMT structure [1] with a thin inner layer is characterized by the high frequency narrow band longitudinal impedance with a single slow propagating $\mathrm{TM}_{01}$ mode at resonant frequency [1]. The frequency dependent longitudinal component of the electric field is given by [1]

$$
E_{z}(\omega)=q Z_{\|}^{0}(\omega)=\frac{j q c Z_{0}}{2 \pi a^{2} \omega_{d}}\left\{\frac{\omega_{1}}{\omega-\omega_{1}}-\frac{\omega_{2}}{\omega-\omega_{2}}\right\},
$$

where $Z_{0}=120 \pi \Omega$ is the impedance of free space, $\omega$ is the frequency, and

$$
\begin{aligned}
\omega_{1,2} & =-j A / 2 \pm \omega_{d}, \quad \omega_{d}=\sqrt{\omega_{0}^{2}-(A / 2)^{2}}, \\
\omega_{0} & =c \sqrt{2 / a d}, \quad A=\frac{2 c}{\sqrt{3} a}\left(\varsigma+\varsigma^{-1}\right), \\
\varsigma & =d \sigma_{1} Z_{0} / \sqrt{3} .
\end{aligned}
$$

Other nonzero components $E_{r}(\omega)$ and $H_{\phi}(\omega)$ are derived from the Maxwell equations 


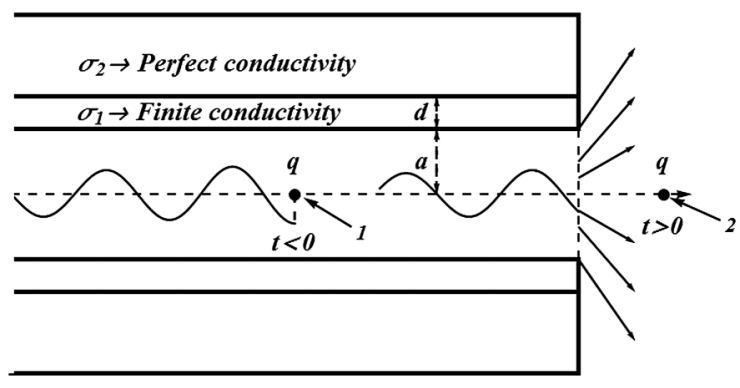

FIG. 1. Geometry of the problem.

$$
E_{r}(\omega)=Z_{0} H_{\phi}(\omega)=j k r E_{z}(\omega) / 2,
$$

where $k=\omega / c$ is wave number.

The radiation begins to propagate in free space at the moment $t=0$ when the particle reaches the aperture section and continues throughout the infinite time interval $0<t<\infty$ (position 2 in Fig. 1). The goal is to obtain the analytical expressions for the radiation field in the free space in the far (relative to the size of the aperture of the waveguide) zone and explore the far field properties.

The exact solution by the Wiener-Hopf method [9] or by the factorization method [10] is not intended to be found out. In this case, it suffices to apply a method similar to the Kirchhoff approximation [7], generalizing it to the case of a nonmonochromatic wave [8]. The above-mentioned method gives qualitatively correct results in the main direction (straight ahead), where it is expected to be the essential part of the radiation.

\section{NEAR FIELD-FAR FIELD TRANSFORMATION ALGORITHM}

The technique of restoring the far field from the known distribution of its tangential electrical components on the plane (near field - far field technique [7]) is used. The algorithm [7] to the extent necessary for the set goals is briefly described and is generalized for the case of non monochromatic (quasimonochromatic) waves.

Let on the plane $z=0$, where the distribution $E_{x, y}^{n}(\omega, x, y)$ of monochromatic electric field components of the radiation is emanating from a source close to it (Fig. 2).

In Fig. 2, the space is described by the Cartesian coordinate system, $X, Y, Z$, the center of which is aligned with the center of the aperture of the waveguide and its $z$ axis is aligned with the symmetry axis of the waveguide. The spherical system of coordinates $R, \theta, \varphi$ describing the far field, and the cylindrical coordinate system $r, \xi, z$ describing the field in the plane $z=0$ and the field propagating in the waveguide are introduced as well.

In the case of a monochromatic light source, the far field at point $\vec{R}(R, \theta, \varphi)$ in a spherical coordinate system is

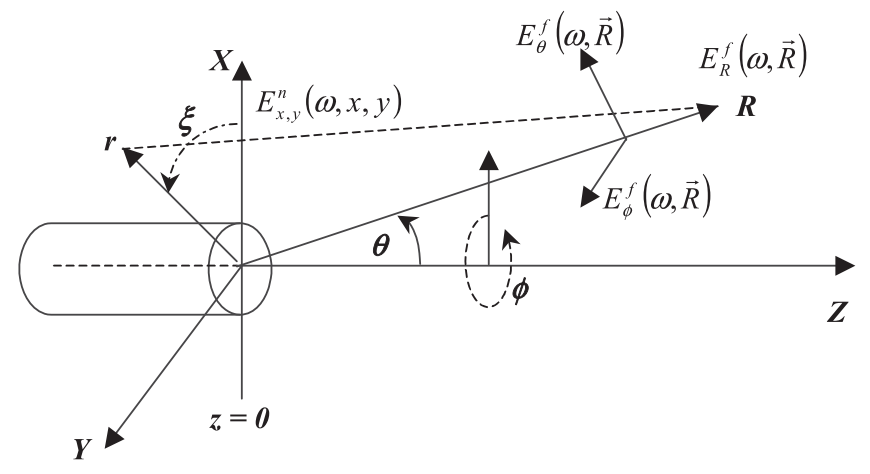

FIG. 2. Near-field—far-field transformation.

associated with the Cartesian components of the near field as follows [7]:

$$
\vec{E}^{f}(\omega, \vec{R})=k \cos \theta \vec{A}\left(k_{x}, k_{y}\right) \frac{e^{i k R}}{R},
$$

where

$$
\begin{aligned}
A_{x, y}\left(k_{x}, k_{y}\right) & =\int_{-\infty}^{\infty} \int_{-\infty}^{\infty} E_{x, y}^{n}(\omega, x, y) e^{-j k_{x} x-j k_{y} y} d x d y, \\
A_{z}\left(k_{x}, k_{y}\right) & =-\frac{1}{k_{z}}\left\{k_{x} A_{x}\left(k_{x}, k_{y}\right)+k_{y} A_{y}\left(k_{x}, k_{y}\right)\right\},
\end{aligned}
$$

with

$$
\begin{aligned}
& k_{x}=k \sin \theta \cos \varphi, \quad k_{y}=k \sin \theta \sin \varphi, \\
& k_{z}=\sqrt{k^{2}-k_{x}^{2}-k_{y}^{2}}=k \cos \theta .
\end{aligned}
$$

The corresponding components in a spherical coordinate system are

$$
\begin{aligned}
& E_{R}=0, \quad E_{\theta}=A_{x} \cos \varphi+A_{y} \sin \varphi, \\
& E_{\varphi}=\cos \theta\left(A_{x} \sin \varphi-A_{y} \cos \varphi\right) .
\end{aligned}
$$

The above equations (4)-(7) illustrate a common near-field-far-field transformation algorithm [7]. If the distribution on the plane is axially symmetric (depends only on the distance $r=\sqrt{x^{2}+y^{2}}$ from the center point of the plane) and is nonzero only within the aperture, the expressions (5) and (7) are simplified and can be represented as follows:

$$
A_{x, y}\left(k_{x}, k_{y}\right)=\int_{0}^{a} \int_{0}^{2 \pi} E_{x, y}^{n}(\omega, r, \xi) e^{-j k r \sin \theta \cos (\varphi-\xi)} d r d \xi,
$$

with

$$
\begin{aligned}
& E_{x}^{n}(\omega, r, \xi)=E_{r}^{n}(\omega, r) \cos \xi \\
& E_{y}^{n}(\omega, r, \xi)=E_{r}^{n}(r, \omega) \sin \xi .
\end{aligned}
$$


Integration in (8) with respect to $\xi$ gives

$$
\left.\begin{array}{l}
A_{x}\left(k_{x}, k_{y}\right) \\
A_{y}\left(k_{x}, k_{y}\right)
\end{array}\right\}=-2 \pi j\left\{\begin{array}{c}
\cos \varphi \\
\sin \varphi
\end{array}\right\} I(\omega, \theta),
$$

with

$$
I(\omega, \theta)=\int_{0}^{a} E_{r}^{n}(\omega, r) J_{1}(k r \sin \theta) r d r .
$$

With regard to (5)-(11) the $z$ component of vector $\vec{A}$ may be represented as

$$
\begin{aligned}
A_{z}\left(k_{x}, k_{y}\right) & =-\operatorname{tg} \theta\left(A_{x}\left(k_{x}, k_{y}\right) \cos \varphi+A_{y}\left(k_{x}, k_{y}\right) \sin \varphi\right) \\
& =2 \pi j I(\omega, \theta) \operatorname{tg} \theta .
\end{aligned}
$$

Spherical coordinates of the vector $\vec{A}$ can be written as follows:

$$
\begin{aligned}
A_{R} & =A_{\varphi}=0, \\
A_{\theta}\left(k_{x}, k_{y}\right) & =\sec \theta\left(A_{x}\left(k_{x}, k_{y}\right) \cos \varphi+A_{y}\left(k_{x}, k_{y}\right) \sin \varphi\right) \\
& =-2 \pi j I(\omega, \theta) \sec \theta .
\end{aligned}
$$

Thus, the single angular spherical far field components of (4) can be written as

$$
E_{\theta}^{f}(\vec{R}, \omega)=Z_{0} H_{\varphi}(\vec{R}, \omega)=-2 \pi j \frac{\omega}{c} I(\omega, \theta) \frac{e^{i \omega(R / c-t)}}{R} .
$$

If the source emits a nonmonochromatic wave, the result (14) must be integrated over all frequencies:

$$
E_{\theta}^{f}(\vec{R})=\int_{-\infty}^{\infty} E_{\theta}^{f}(\vec{R}, \omega) d \omega .
$$

The radial component of the electric field of the wave incident on the external aperture of the waveguide, formed by the wakefield radiation of the point ultrarelativistic charged particle, moving along the axis of the described two-layer waveguide, has a radial dependence proportional to $r$ (3). Thus, the integral (11) can be calculated explicitly:

$$
I_{\infty}(\omega, \theta)=\frac{j c q Z_{0}}{4 \pi \omega_{d}}\left\{\frac{\omega_{1}}{\omega-\omega_{1}}-\frac{\omega_{2}}{\omega-\omega_{2}}\right\} \frac{J_{2}(k a \sin \theta)}{k \sin \theta},
$$

where $J_{2}(x)$ is the Bessel function of the first kind and second order [11], and the spectral-angular distribution of the far field may be written as

$$
\begin{aligned}
E_{\theta}^{f}(\vec{R}, \omega)= & \frac{q Z_{0}}{2 \omega_{d}}\left(\frac{\omega \omega_{1}}{\omega-\omega_{1}}-\frac{\omega \omega_{2}}{\omega-\omega_{2}}\right) \\
& \times \frac{J_{2}(k a \sin \theta)}{\sin \theta} \frac{e^{j \omega(R / c-t)}}{R} .
\end{aligned}
$$

The total field in the far zone at a point $\vec{R}(R, \theta, \varphi)$ in the integral form of (15) can be written as follows:

$$
\begin{aligned}
E_{\theta}^{f}(\vec{R})= & \frac{q Z_{0}}{2 \omega_{d}} \int_{-\infty}^{\infty}\left(\frac{\omega \omega_{1}}{\omega-\omega_{1}}-\frac{\omega \omega_{2}}{\omega-\omega_{2}}\right) \\
& \times \frac{J_{2}(k a \sin \theta)}{\sin \theta} \frac{e^{j \omega / c s}}{R} d \omega,
\end{aligned}
$$

with $s=R-c t$.

The integral in (18) is calculated using the analytic continuation of the integrand to the complex plane of the frequencies $\omega$. The integrand in (18) has two poles $\omega=\omega_{1,2}$ (2), located in the lower part of the complex plane. During the calculation, the asymptotic form of the Bessel function $J_{2}(x)$ in (18), valid for large arguments (see, for example, [11]) should be taken into account:

$$
\begin{aligned}
J_{2}(x) & =-\sqrt{\frac{1}{\pi x}}\left\{\frac{1-j}{2} e^{i x}+\frac{1+j}{2} e^{-i x}\right\}, \quad|x| \rightarrow \infty, \\
x & =\frac{\omega}{c} a \sin \theta, \quad 0<\theta \leq \frac{\pi}{2} .
\end{aligned}
$$

The exponential factors in the integrand in (18) in that case take the form $e^{j \frac{\omega}{c}(a \sin \theta+R-c t)}$ and $e^{j \frac{\omega}{c}(-a \sin \theta+R-c t)}$ for the first and second terms in (19), respectively. In the integration along the contour in the shape of a semicircle with infinite radius, located in the lower half $(\operatorname{Im} \omega<0,|\omega| \rightarrow \infty)$, the first and second terms vanish under the conditions $c t>R+a \sin \theta$ and $c t>R-a \sin \theta$, respectively. So, both of these terms are vanishing if $c t>R+a \sin \theta$. Under this condition, the integration in (18) is reduced to the summation of the residue formed by the poles $\omega_{1,2}(2)$ :

$$
E_{\theta}^{f}(\vec{R})=\frac{\pi Z_{0}}{R}\left(B\left(\theta, \omega_{1}\right) e^{j \omega_{1}(R / c-t)}+B\left(\theta, \omega_{2}\right) e^{j \omega_{2}(R / c-t)}\right),
$$

where

$$
\begin{aligned}
B(\theta, \omega) & =j \frac{\omega^{2}}{\omega_{d}} J_{2}\left(\frac{\omega}{c} a \sin \theta\right) / \sin \theta, \\
B\left(\theta, \omega_{2}\right) & =B^{*}\left(\theta, \omega_{1}\right), \quad c t>R+a \sin \theta .
\end{aligned}
$$

In the upper half $(\operatorname{Im} \omega>0,|\omega| \rightarrow \infty)$, the first term of the integrand in (18) tends to zero under condition $c t<R+a \sin \theta$, while the second one vanishes when $c t<R-a \sin \theta$. The integration along the semicircle in the upper half is possible upon vanishing of both terms in infinity, i.e., when $c t<R-a \sin \theta$. Under this condition, due to the absence of poles in the upper half-plane, the integral (18) vanishes:

$$
E_{\theta}^{f}(\vec{R})=0, \quad c t<R-a \sin \theta .
$$


The value of the integral (18) in the space-time domain $R+a \sin \theta>c t>R-a \sin \theta$ stays uncertain. To determine the value of the integral (18) in this space-time domain, we represent it in the form of a sum of two integrals:

$$
E_{\theta}^{f}(\vec{R})=I_{1}(\vec{R})+I_{2}(\vec{R}),
$$

where

$$
\begin{aligned}
I_{1,2}(\vec{R})= & \frac{q Z_{0}}{2 \omega_{d}} \int_{-\infty}^{\infty}\left(\frac{\omega \omega_{1}}{\omega-\omega_{1}}-\frac{\omega \omega_{2}}{\omega-\omega_{2}}\right) \\
& \times \frac{Q_{1,2}(k a \sin \theta)}{\sin \theta} \frac{e^{j \omega / c s}}{R} d \omega,
\end{aligned}
$$

with

$$
\begin{aligned}
Q_{1,2}(\theta, \omega)= & \frac{1}{2}\left\{J_{2}\left(\frac{\omega}{c} a \sin \theta\right) \mp j \mathrm{H}_{2}\left(\frac{\omega}{c} a \sin \theta\right)\right. \\
& \left. \pm j \frac{2 \omega a \sin \theta}{3 \pi c}\right\},
\end{aligned}
$$

where $\mathrm{H}_{2}(z)$ is the Struve function [11].
The asymptotic representations of functions $Q_{1}$ and $Q_{2}$, which are valid for $|\omega / c a \sin \theta| \rightarrow \infty$, are given by

$$
Q_{1,2}(\theta, \omega)=-\frac{1 \pm j}{2 \sqrt{\pi \omega / c a \sin \theta}} e^{\mp j \omega / c a \sin \theta} .
$$

Thus, in the integration along the contour in the shape of a semicircle with infinite radius, located in the lower half $(\operatorname{Im} \omega<0,|\omega| \rightarrow \infty)$, the exponential term in integral $I_{1}$ vanishes under the condition $c t>R-a \sin \theta$ and in the integration along the contour in the same shape, located in the bottom half $(\operatorname{Im} \omega>0,|\omega| \rightarrow \infty)$, the exponential term in integral $I_{2}$ vanishes under the conditions $c t<R+a \sin \theta$. Thus, in the total space-time interval it is possible to achieve the simultaneous integration of integral $I_{1}$ in the lower half-plane and of the integral $I_{2}$ on the upper half-plane defined by the inequality $R-a \sin \theta<c t<R+a \sin \theta$. In this interval the value of integral $I_{1}$ is determined by the poles, located in the lower half-plane, and the integral $I_{2}$, vanishes due to the absence of poles in the upper half-plane. Thus, the far field radiated by the open end of the waveguide in the entire space-time interval $-\infty<c t<\infty$ has been determined:

$$
E_{\theta}^{f}(\vec{R})= \begin{cases}\frac{\pi Z_{0}}{R} e^{\frac{A}{2}(R / c-t)}\left\{B\left(\theta, \omega_{1}\right) e^{j \omega_{d}(R / c-t)}+\text { c.c. }\right\}, & c t>a \sin \theta+R \\ \frac{\pi Z_{0}}{R} e^{\frac{A}{2}(R / c-t)}\left\{B_{1}\left(\theta, \omega_{1}\right) e^{j \omega_{d}(R / c-t)}+\text { c.c. }\right\}, & -a \sin \theta+R<c t<a \sin \theta+R, \\ 0, & c t<-a \sin \theta+R,\end{cases}
$$

where c.c. denotes the complex conjugation, and $B_{1}\left(\theta, \omega_{1}\right)$ is given by

$$
\begin{aligned}
B_{1}(\theta, \omega) & =j \frac{\omega^{2}}{\omega_{d}} Q_{1}(\theta, \omega) / \sin \theta, \\
B_{1}\left(\theta, \omega_{2}\right) & =B_{1}{ }^{*}\left(\theta, \omega_{1}\right) .
\end{aligned}
$$

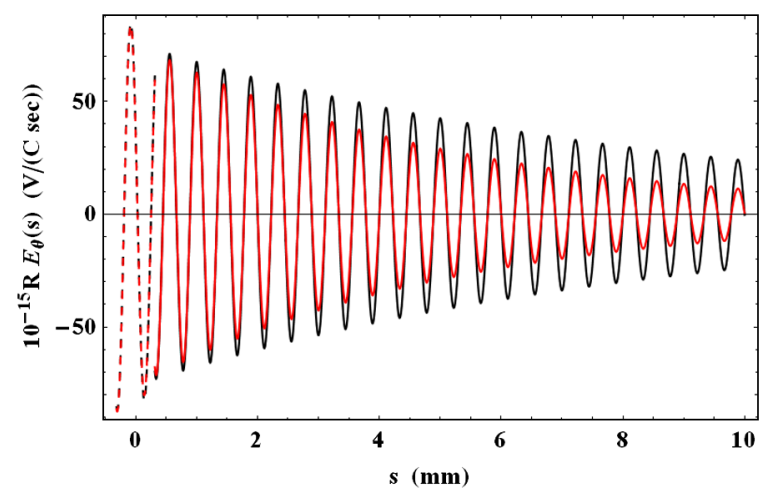

Thus, according to (27), the field is formed by two successive radiation pulses. The first impulse, that reaches the observation point, is formed in a finite time interval $-a \sin \theta+R<c t<a \sin \theta+R$. The second pulse comprehends the observation point in time moment $t=$ $a \sin \theta+R$ and lasts until $t=\infty$. The duration of the first pulse is conditioned by the size of radius of the waveguide and by the value of the viewing angle and reduces with a

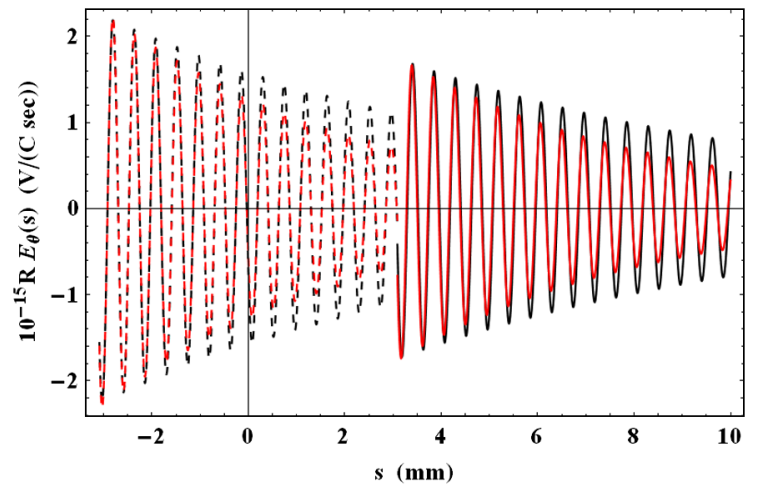

FIG. 3. Spatial-temporal distribution of the radiation field at fixed angles of observation; $\theta=\pi / 100$ (left), $\theta=\pi / 10$ (right); first pulse (dotted line), second pulse (solid line); $\varsigma=1$ (black line), $\varsigma=3$ or $\varsigma=1 / 3$ (red line). 
decrease of the angle of observation. Since the radiation is narrowly targeted, the latter's role in shaping the overall flow of radiation should be small. Figure 3 shows the comparison of the space-time distribution for small (left) and relatively large (right) viewing angles.

Spatial-temporal frequency of the pulses is equal to $\omega_{d}$ (for $d \ll a \omega_{d} \approx \omega_{0}$ ). The damping is determined by the attenuation coefficient $A$. The minimum value of the attenuation coefficient accounts for $\varsigma=1$. Note, that according to (2), the attenuation coefficient $A$ does not change with the transition from $\varsigma$ to $\varsigma^{-1}$, since the curves for $\varsigma=3$ and $\varsigma=1 / 3$ are identical.

\section{INTERPRETATION BY THE HELP OF DIFFRACTION PHENOMENON}

The presence of two consecutive pulses can be explained with the help of the diffraction phenomenon. Substituting asymptotic expressions (19) into (21) and using the result for the upper row of (27), one gets for the space-time domain $c t>a \sin \theta+R$ :

$$
E_{\theta}^{f}(\vec{R})=-\frac{\pi Z_{0}}{R \sin \theta} \sqrt{\frac{1}{\pi k a \sin \theta}} e^{-\frac{A}{2} t}\left\{j \frac{\omega_{1}^{2}}{\omega_{d}}\left(\frac{1-j}{2} e^{\left(\frac{A}{2 c}+j \frac{\omega_{d}}{c}\right) s_{+}}+\frac{1+j}{2} e^{\left(\frac{A}{2 c}+j \frac{\omega_{d}}{c}\right) s_{-}}\right) e^{-j \omega_{d} t}+\text { c.c. }\right\}
$$

with $s_{ \pm}= \pm a \sin \theta+R$. Similarly, using (25), (28), and (27), one obtains an asymptotic expression for the first pulse [the second line in (27)]:

$$
\begin{aligned}
E_{\theta}^{f}(\vec{R})= & -\frac{\pi Z_{0}}{R \sin \theta} \sqrt{\frac{1}{\pi k a \sin \theta}} \\
& \times e^{-\frac{A}{2} t}\left\{j \frac{\omega_{1}^{2}}{\omega_{d}} \frac{1+j}{2} e^{\left(\frac{A}{2 c}+j \frac{\omega_{d}}{c}\right) s_{-}} e^{-j \omega_{d} t}+\text { c.c. }\right\} .
\end{aligned}
$$

As it is seen from (29) and (30), these high-frequency asymptotic representations of fields formed by diffraction beams emanate from the edge of the waveguide opening. These rays correspond to the partial quasimonochromatic fields with a frequency of $\omega_{d}$ and decreasing amplitude, with a damping factor $A$. The field of the first pulse (30) is formed by a single beam emanating from a point on the edge of the waveguide cross section closest to the point of observation $\left(s_{-}\right.$is the distance from this point to the observation point). In the formation of the field of the second (main) pulse, the ray from the edge of the most distant point of the cross section of the waveguide $\left(s_{+}\right.$is a distance from this point to the observation point) is also involved. One observation point and two diffraction points are contained in the plane of the main longitudinal cross section of the waveguide, which contains the axis of the waveguide.

When considering the radiation of monochromatic waves without a fixed initial time point of emission (steady state process) and with the zero damping decrement $[10,12,13]$, the process of interference of the two diffracted rays is fully described by the help of their geometric eikonals (geometric distances $s_{ \pm}$between the diffraction
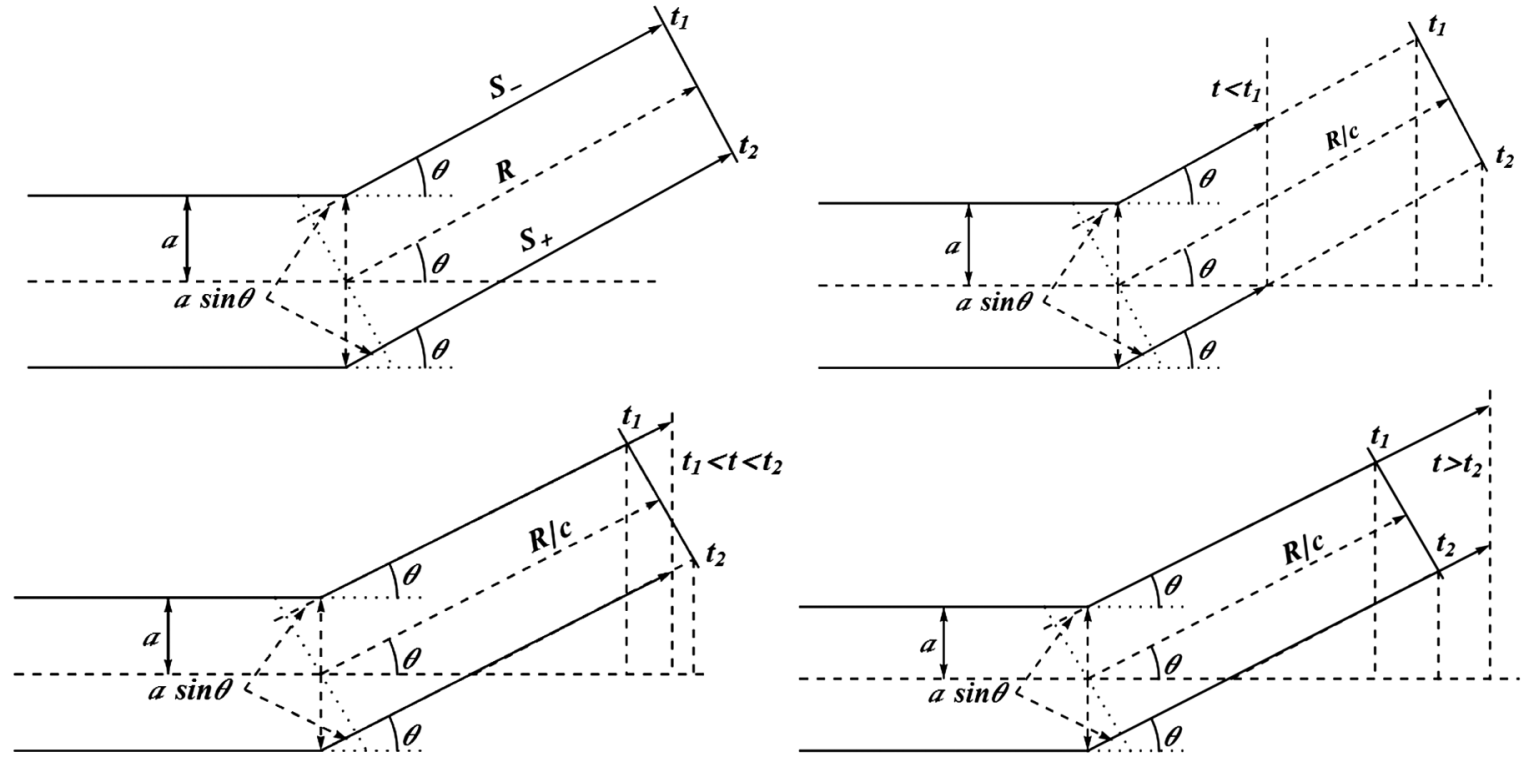

FIG. 4. Diffraction at the open end of the waveguide: steady-state regime (top left); dynamic regime: $t<t_{1}$ (top right); $t_{1}<t<t_{2}$ (bottom left); $t>t_{2}$ (bottom right). 
points and the point of observation). As an example of diffraction rays of an axially symmetric mode on the open end of a perfectly conducting round waveguide is presented in Fig. 4 (top left) schematically.

In our case, the open end of the waveguide begins to radiate when the particle reaches its cross section, i.e., at time $t=0$. The ray from the near point reaches the point of observation, during time $t_{1}=s_{-} / c$, and the ray from the far point, respectively, during time $t_{2}=s_{+} / c$. Thus, the rays torn from the edge reach the observation point at time $t_{1}^{\prime}=t-t_{1}$ and at $t_{2}^{\prime}=t-t_{2}$, respectively. If $t<t_{1}$, then $t_{1}^{\prime}<0, t_{2}^{\prime}<0$ and neither of the two rays cannot reach the point of observation (Fig. 4, top right), which corresponds to the zero field therein. Consequently, during the time interval $t_{1}<t<t_{2}$, one has $t_{1}^{\prime}>0, t_{2}^{\prime}<0$ (Fig. 4, bottom left) and only the nearest beam can reach the observation point. Later on (at $t>t_{2}$, then $t_{1}^{\prime}>0, t_{2}^{\prime}>0$ ), the second ray also has enough time to arise and comes to the observation point. In this case the field is formed as a result of the interference interaction of the two rays (Fig. 4, bottom right).

As can be seen from Fig. 4, in the case of the dynamic regime only, the beam crosses the time line $t_{1}-t_{2}$ and makes a contribution to the radiation, while in the steadystate regime both rays touch this time line at the same time. The concept of two successive pulses is consistent with the principle of causality and is interpreted with the help of the time lag phenomenon.

A dual-beam radiation pattern is formed gradually, starting from the angles $\theta=0$ up to $\theta=\pi / 2$ in the time interval $R<c t<R+a$. Thus, the total time of the formation of a two-beam radiation is $c t=R+a$. In the time interval $R+a<c t<\infty$, where the radiation over the entire range of angles has a continuous periodic monotonically decreasing character and is described by the first line of (29), and for $A \ll \omega_{0}=c \sqrt{2 / a d}$ (or $d \ll a$ ), its radiation pattern can be identified, independent of the time (Fig. 5), as

$$
F(x)=\frac{J_{2}(x)}{x}, \quad x=\frac{\omega_{0}}{c} a \sin \theta .
$$

The maximum of the diagram (Fig. 4) does not depend on the value of the conductivity of the inner cover and can be determined by the following formula:

$$
\theta_{\max }=\operatorname{ArcSin}(2.3 \sqrt{d / 2 a}) \approx 2.3 \sqrt{d / 2 a} .
$$

For $d=1 \mu \mathrm{m}: \theta_{\max }=23 \mathrm{mrad}$ for $a=1 \mathrm{~cm}$ and $\theta_{\max }=73 \mathrm{mrad}$ for $a=1 \mathrm{~mm}$.

The radiation power of the main lobe is concentrated in the range of angles $0<\theta<2 \theta_{\max }$. It constitutes $\sim 79 \%$ of the total power radiated in the front half-space, regardless of the values of the parameters $a$ and $d$ (provided that $d \ll a$ ).

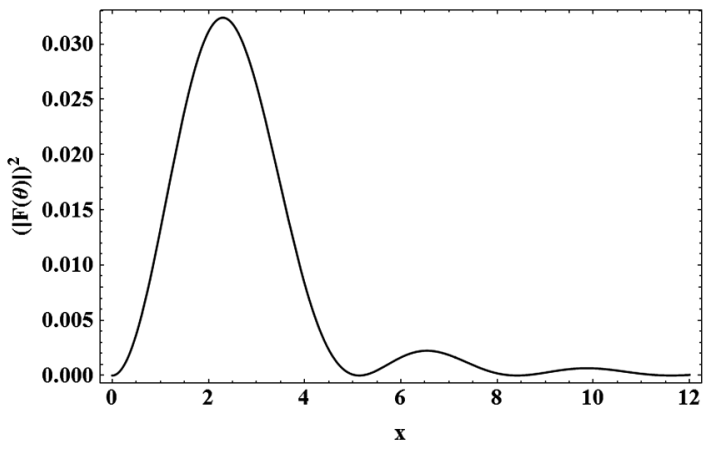

FIG. 5. The radiation pattern in power (at $c t>R+a$ ).

\section{ENERGY DENSITY ANGULAR DISTRIBUTION}

The most obvious way to fix the emission and detection of directional properties of the field is the exposure of film (located at some distance from the aperture $R \gg a$ parallel to the aperture), which captures the power that falls on it during the entire time of radiation. In this case, it may be difficult to select the components of radiation, which corresponds to the first or the second pulse.

In order to evaluate the easily measurable integral characteristics of the radiation and its directional properties, it is necessary to determine the angular distribution of the energy density of the field emitted during the entire time of radiation. Since the emitted pulses are separated in time and space, it is possible to perform a separate calculation of energy density distribution for each of the pulses, and to determine the percentage of each one of them. Thus, the angular distributions of the energies density of the first $U_{1}(\theta)$ and second $U_{2}(\theta)$ pulses can be written with the help of the expressions for the field emission in the following way:

$$
\begin{aligned}
U_{1}(\theta) & =\frac{2 \pi^{2} q^{2} Z_{0}}{c R^{2}}\left|B_{1}\left(\theta, \omega_{1}\right)\right|^{2} \int_{-a \sin \theta}^{a \sin \theta} e^{-A / c s} d s \\
& =\frac{4 \pi^{2} q^{2} Z_{0}}{R^{2}}\left|B_{1}\left(\theta, \omega_{1}\right)\right|^{2} \frac{\operatorname{Sinh}(A a / c \sin \theta)}{A}, \\
U_{2}(\theta) & =\frac{2 \pi^{2} q^{2} Z_{0}}{c R^{2}}\left|B\left(\theta, \omega_{1}\right)\right|^{2} \int_{a \sin \theta}^{\infty} e^{-A / c s} d s \\
& =\frac{2 \pi^{2} q^{2} Z_{0}}{R^{2}}\left|B\left(\theta, \omega_{1}\right)\right|^{2} \frac{e^{-A / c a \sin \theta}}{A}
\end{aligned}
$$

whereas the total energy density is the sum of both densities:

$$
U(\theta)=U_{1}(\theta)+U_{2}(\theta) .
$$

The direction and the overall shape of the total density of the angular distribution of the radiation energy is mainly determined by the second (main) pulse of radiation (Fig. 6). 

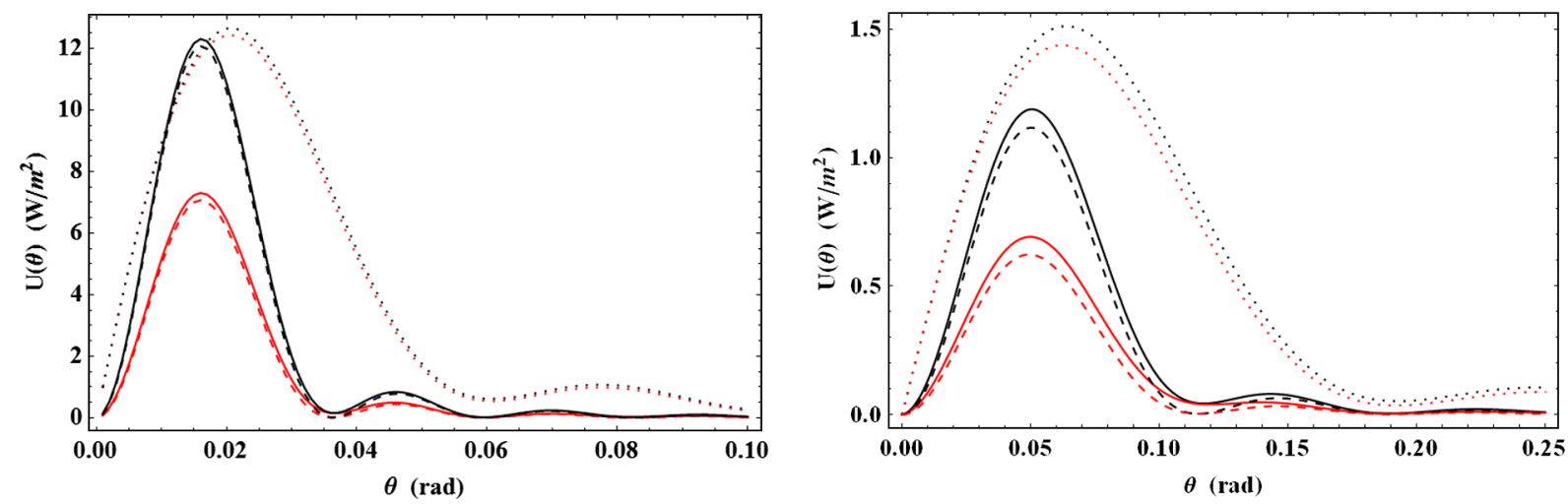

FIG. 6. The angular distribution of the radiation energy $(q=0.1 \mathrm{nC}, R=1 \mathrm{~m}) ; a=1 \mathrm{~cm}$ (left), $a=1 \mathrm{~mm}$ (right); total density distribution (solid line); second pulse contribution (dashed line); first pulse contribution (dotted line); $\varsigma=1$ (black line); $\varsigma=3$ (red line). The scale of the distribution curve for the first pulse is increased by 50 times for $a=1 \mathrm{~cm}$ (left) and by 20 times for $a=1 \mathrm{~mm}$ (right).

However, as can be seen from Fig. 6, the relative contribution of the first pulse in the radiation increases with increasing deviation of the parameter $\varsigma$ from its optimal value $\varsigma=1$.

A decrease in the radius of the waveguide leads to a general broadening of the energy diagram, and its main lobe, in particular (in agreement with the general theory of antennas [14]), to the simultaneous increase of the contribution of the first pulse. The angle of orientation of the main peak of the energy diagram is still due to the first maximum of the function $J_{2}\left(\omega_{0} / c a \sin \theta\right) / \sin \theta$ (under condition $A \ll \omega_{0}$, which is in the frame of our consideration) and can be defined by the formula (32).

As shown in Fig. 7, the ratio of the contributions from the first and second pulses depends strongly on the value of $\varsigma$. A quantitative assessment of the relative contribution of the primary (formed) radiation relative to the total flux of the radiation produced by calculating their ratio for different values of $\varsigma$ is

$$
\eta=U_{2}(\theta) /\left[U_{1}(\theta)+U_{2}(\theta)\right] .
$$

A graph of $\eta$ versus $\varsigma$ is shown in Fig. 7 .

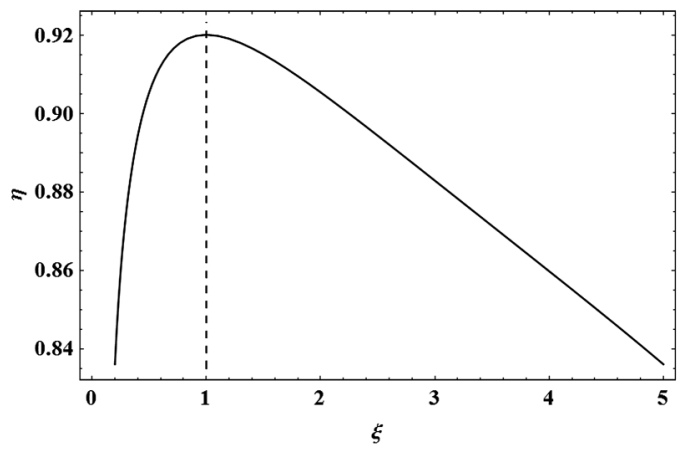

FIG. 7. The relative contribution of power, formed by the second pulse in the total output power depending on the parameter $\varsigma$.
The variation of the parameter $\varsigma$ from 0.25 to 5 reduces the contribution of the main emission by $8 \%$, which did not significantly affect the qualitative nature of the radiation. Selection of the optimum value of the parameter $\varsigma=1$ is important, however, because the deviation from it results in attenuation of the radiation energy (Fig. 7) and its high damping (Fig. 3). Selection of the optimal parameter value $\varsigma$ is associated with the choice of the agreed value of the lower layer thickness $d$ and its conductivity $\sigma_{1}$ (2). In particular, when $d=1 \mu \mathrm{m}$, the optimum value of the conductivity is equal to $4.6 \times 10^{3} \Omega^{-1} \mathrm{~m}^{-1}$.

\section{CONCLUSION}

The main outcome of the research, apparently, is the demonstration of the possibility of generating a focused and narrow-band radiation based on wakefield radiation in the two-layer metallic waveguide. Most of the flux is concentrated within a narrow conical surface with halfangle $\theta_{\max }$. The analytic formulas describing the radiation field at various stages of its formation are obtained. The process of the formation of radiation is considered in detail and the space-time region of its formation is determined. The theory of two successive pulses forming radiation is developed with its further geometric justification.

[1] M. Ivanyan, A. Grigoryan, A. Tsakanian, and V. Tsakanov, Phys. Rev. ST Accel. Beams 17, 021302 (2014).

[2] J. B. Rosenzweig, G. Andonian, P. Muggli, P. Niknejadi, G. Travish, O. Williams, K. Xuan, V. Yakimenko, S. H. Gold, and G.S. Nusinovich, AIP Conf. Proc. 1299, 364 (2010).

[3] G. Stupakov and K. L. F. Bane, Phys. Rev. ST Accel. Beams 15, 124401 (2012).

[4] G. V. Sotnikov, T. C. Marshall, and J. L. Hirshfield, Phys. Rev. ST Accel. Beams 12, 061302 (2009). 
[5] S. S. Baturin, I. L. Sheinman, A. M. Altmark, D. A. Semikin, and A. D. Kanareykin, Tech. Phys. Lett. 37, 394 (2011).

[6] B. Grigoryan et al., Proceedings of the 2nd International Particle Accelerator Conference, San Sebastián, Spain (EPS-AG, Spain, 2011), pp. 1066-1068.

[7] E. B. Joy and D. T. Paris, IEEE Trans. Antennas Propag. 20, 253 (1972).

[8] M. Born and E. Wolf, Principles of Optics (Pergamon, Oxford, 1980), 6th ed.

[9] R. Mittra and S. W. Lee, Analytical Techniques in the Theory of Guided Waves (Macmillan, New York, 1971).
[10] L. A. Vainshtein, The Theory of Diffraction and the Factorization Method (Sovetskoe Radio, Moscow, 1966) (in Russian).

[11] M. Abramovitz and A. Stegun, Handbook of Mathematical Functions (National Bureau of Standards, Washington, DC, 1964).

[12] H. Y. Yee, L. B. Felsen, and J. B. Keller, SIAM J. Appl. Math. 16, 268 (1968).

[13] M. I. Ivanian, Radiotekhnika i electronica 27, 665 (1982) (in Russian).

[14] R. Dean Straw, The ARRL Antenna Book (American Radio Relay League, Newington, CT, 2005), 20th ed. 